
\title{
25 Research Soure \\ Influencing factors of aggressive behavior among left-behind junior high school students: structural equation model
}

\section{Yi-Fei Li}

Central South University Xiangya School of Nursing

Jie Zhang

Central South University Xiangya School of Nursing

Juan Li

Central South University Xiangya School of Nursing

Yi-Ping Chen

Central South University Xiangya School of Nursing

Si-Lan Yang

Central South University Xiangya School of Nursing

Ying-Xiang Tao

Central South University Xiangya School of Nursing

Bi-Yun Ye

Central South University Xiangya School of Nursing

Jing-Ping Zhang

Central South University Xiangya School of Nursing

Ting Mao ( $\nabla$ csumaoting@163.com )

Central South University Xiangya School of Nursing

\section{Research}

Keywords: left-behind junior high school students, aggressive behavior, life events, self-esteem, resilience, coping style, structural equation model

Posted Date: April 29th, 2020

DOI: https://doi.org/10.21203/rs.3.rs-25120/v1

License: (c) (1) This work is licensed under a Creative Commons Attribution 4.0 International License.

Read Full License 


\section{Abstract \\ Background}

High aggressive behavior will harm the physical and mental health of children, and the probability of aggressive behaviors among left-behind junior high school students is significantly higher than that of non-left-behind ones, but the systematic research and intervention measures concerning the aggressive behavior of the left-behind junior high school students quite deficient at present. This study adopts the structural equation model to analyze the interrelationship and action modes of various factors affecting the aggressive behavior of the left-behind junior high school students to provide scientific basis for the mitigation and prevention of their aggressive behavior.

\section{Methods}

A structural equation model was constructed based on data from a cross-sectional survey of 751 leftbehind junior high school students using Adolescent Self-rating Life Events Checklist, Rosenberg SelfEsteem Scale, Resilience Scale for Chinese Adolescents, Coping Style Questionnaire, and Buss-Warren Aggression Questionnaire.

\section{Results}

Self-esteem, resilience, positive coping, negative coping and household income have different direct or indirect effects on the aggressive behavior of left-behind junior high school students. Among them, selfesteem, resilience, positive coping and negative coping are the important mediating factors between life events and aggressive behavior of left-behind junior high school students.

\section{Conclusions}

Parents, teachers and temporary guardians in the care of left-behind children should try to avoid possible adverse effects that the life events have on the children, meanwhile should also through effective psychological intervention and social support, improving the self-esteem, psychological resilience and positive coping tendencies of left-behind children, in order to reduce or prevent their aggressive behaviors.

\section{Background}

With the rapid development of China's economy and the acceleration of urbanization, the barriers of population flow between urban and rural areas are broken, and more and more rural people are moving to cities ${ }^{[1]}$. According to the latest data released by China's National Bureau of Statistics in 2019, the total number of migrant workers in China has reached nearly 300 million, of which 170-million people are from 
other places, accounting for more than half of the total ${ }^{[2]}$. However, due to the establishment of the Dual Hukou System in China, some migrant workers are restricted by many factors such as household registration, economy and urban school entrance requirements, and have to leave their minor children in rural homes, thus forming a special group of children: left-behind children ${ }^{[3,4]}$.

Left-behind children refer to children aged 6 to 16 who are in the stage of compulsory education and are left to be cared for or live alone in the place of their domicile after their parents go out to work ${ }^{[5]}$. According to the latest data released by the Ministry of Education in 2018, the number of left-behind children in rural China has exceeded 15 million ${ }^{[6]}$. 91.3\% have suffered different levels of mental, physical, sexual or neglect violence from their families or schools ${ }^{[7]}$. As a result, left-behind children have higher levels of anxiety, depression, suicidal tendencies and aggressive behavior in terms of mental health compared with children from normal families ${ }^{[8-12]}$, and they are more prone to violent crimes and aggressive behavior in terms of problem behaviors ${ }^{[13]}$. Meanwhile, adolescence is an important stage in the development of individual personality, sociality and values, during which rebellious and aggressive behaviors are particularly prominent ${ }^{[14]}$. Under the same frustration situation, the probability of aggressive behaviors among left-behind junior high school students is significantly higher than that of non-left-behind ones ${ }^{[3,5,14]}$.

In addition to the social harmfulness, aggressive behavior may also have a negative impact on the physical and mental development of individuals. Research shows that teens with high aggressiveness are more likely to have psychological problems, self-harm, suicide and commit violent crimes as adults [15-17]. However, the systematic research and intervention measures concerning the aggressive behavior of the left-behind junior high school students are quite deficient at present. Most studies only analyzed the effect of a single factor, and could not judge the direct and indirect effects among the factors ${ }^{[18,19]}$. Therefore, this study adopts the structural equation model to analyze the interrelationship and action modes of various factors affecting the aggressive behavior of the left-behind junior high school students to provide scientific basis for the mitigation and prevention of the aggressive behavior of left-behind junior high school students.

\section{Stress-coping Model Of Left-behind Junior High School Students}

Left-behind junior high school students are more vulnerable to psychological stress, negative coping and aggressive behavior ${ }^{[20,21]}$. The most widely used theoretical model to guide these studies is the cognitive-phenomenological-transactional theoretical (CPT) model ${ }^{[22]}$. This model is derived from Lazarus and Folkman's stress and coping theory and emphasizes the absolute role of cognitive evaluation in the process of stress and coping ${ }^{[23]}$, which is consistent with the "frustration-aggression" theory proposed by Miller, and regarded as the first classical theory to study human aggressive behavior. Based on "frustration-aggression" theory, aggressive behavior is one kind of behavioral response to 
human frustration under a certain situation ${ }^{[24]}$. This process is consistent with the three continuous variables in the implementation of the CPT theoretical model: stressors, environmental information, and individual-environmental effects ${ }^{[22]}$.

The initial stressors are various life events faced by children ${ }^{[25]}$, among which have a significant impact on left-behind junior high school students, like interpersonal relationship, learning pressure, punishment, loss and health adjustment ${ }^{[26]}$. Intermediate stage, namely environmental information, refers to the evaluation method of the relationship between the individual and the environment, including the primary evaluation, secondary evaluation and reevaluation of individuals on the environment ${ }^{[22]}$. This stage determines the occurrence and form of stress, which reflects the role of individual cognition and its influencing factors in the process of stress coping. The final effect may be negative or positive ${ }^{[22]}$. However, due to the lack of family support including timely care and help from their parents, left-behind children are more inclined to adopt negative coping methods ${ }^{[20]}$, such as aggressive behaviors ${ }^{[14]}$.

Therefore, in the stress response model, it is essential to investigate the major life events confronted by the left-behind junior high school students and to explore the key factors that may have an impact on the cognition and evaluation of the left-behind junior high school students, which is very important to reduce and prevent the aggressive behavior of the left-behind junior high school students.

\section{Self-esteem, resilience and coping style as mediators in the stress-coping model}

Although many scholars ${ }^{[14,20,27]}$ have analyzed and studied the aggressive behavior of left-behind junior high school students under the guidance of the "frustration-aggression" theory and the stress process models, most studies did not consider the impact of life events or only used factor analysis and correlation analysis to determine the direct or indirect effects of the factors ${ }^{[18]}$. Studies have found that adolescent's aggressive behavior is significantly correlated to academic, interpersonal, environmental, self-conscious and other life events ${ }^{[27]}$.

Apart from life events, a large number of theories and studies have concluded that cognition and evaluation of frustration are more important in frustration responses ${ }^{[28]}$, and are mainly reflected in the three aspects of mood, self-esteem and personality traits ${ }^{[27]}$. Berkowitz mentioned that frustration can awaken the individual emotions in his revision of "frustration-aggression theory" [29], and different individuals may have positive or negative emotional reactions ${ }^{[30]}$. Specifically speaking, positive emotions are more likely to produce positive responses such as humor and sublimation, while negative emotions are more likely to lead to negative responses such as indifference and avoidance ${ }^{[30]}$. Furthermore, the positive response of middle school students was significantly negatively correlated with aggressive behavior, while the negative response was significantly positively correlated with aggressive behavior ${ }^{[31]}$. In the same setbacks, high-esteem teens were more likely to experience proactive aggression and low-esteem teens were more likely to produce reactive aggression ${ }^{[32,33]}$. Resilience refers to the dynamic process in which an individual can still adapt well in a dangerous environment ${ }^{[34]}$, which is 
significantly positively correlated with the individual's positive personality traits ${ }^{[35]}$. Junior high school students with high levels of resilience are more likely to control and stabilize their emotions after a setback, thus reducing the possibility of the occurrence of aggressive behavior ${ }^{[36]}$. Therefore, although it has not been studied in the comprehensive model, self-esteem, resilience and coping style probably important intermediary factors for the life events and aggressive behavior choices of left-behind junior high school students.

\section{Relationship among self-esteem, resilience and coping style and their different effects}

Some retrospective studies on self-esteem, resilience and coping style of left-behind junior high school students can be found that there is some correlation among them. Studies have shown that individuals with high levels of resilience and self-esteem are more likely to perceive positive external stimuli and choose to respond positively ${ }^{[32,36]}$, while there is also a significant positive correlation between selfesteem and resilience ${ }^{[37]}$. Therefore, we can assume the relationship among self-esteem, resilience and coping style in the same frustration situation, that is: self-esteem and resilience are positively correlated with positive coping, and are negatively correlated with negative coping, self-esteem have positive associations with resilience, and negative coping negatively the positive coping.

Besides, whether self-esteem, resilience, coping styles and aggressive behavior have different associations with adaptation outcomes also warrants attention, as this may be helpful to formulate more targeted intervention strategies. Only one study ${ }^{[38]}$ has examined both resilience and coping styles in analyzing adolescent aggressive behavior, in which it found that both resilience and coping style can negatively predict the adolescent's aggressive behavior, and the coping style can also influence the adolescent's aggressive behavior through the mediating effect of resilience. What's more, Wang's study

${ }^{[39]}$ has examined both self-esteem and coping styles in analyzing adolescent aggressive behavior, it found that self-esteem and coping styles could predict adolescent aggressive behavior, and that coping styles did not as mediators between self-esteem and aggressive behavior. However, there is no research to confirm whether self-esteem has an intermediary effect between coping styles and aggressive behaviors, and whether self-esteem, resilience, negative coping and positive coping have mediating effects in the process of life events stimulating aggressive behaviors. Therefore, although self-esteem, resilience and coping style can predict the aggressive behavior of adolescents, their specific effects and modes of action need to be further explored.

\section{The Aim Of The Present Study}

Based on our literature review, there is a lack of comprehensive comparison and analysis on the direct and indirect influencing factors of left-behind junior high school students' aggressive behavior at present. The comprehensive analysis of the interaction of the influencing factors is very important for the development and formulation of targeted or corresponding programs to reduce the aggressive behavior of left-behind junior high school students and promote their physical and mental health. Therefore, taking 
important background variables into account within the stress-coping model, we selected left-behind junior high school student's life events as stressors, self-esteem, resilience and coping style as intermediary variables, aggressive behavior as outcome to formulate a hypothetical model A(Fig. 1), to facilitate testing and clarification of the following hypotheses:

H1: General information, life events, self-esteem, psychological flexibility and coping style may have different effects on the aggressive behavior of left-behind junior high school students.

$\mathrm{H} 2$ : self-esteem, resilience and coping style may be mediators between left-behind junior high school student's life events and aggressive behavior.

H3: resilience may be mediators between positive coping and aggressive behavior(3a), resilience, positive coping may be mediators between negative coping and aggressive behavior(3b), positive coping, negative coping may be mediators between self-esteem and resilience (3c), positive coping may be mediators between negative coping and resilience(3d).

\section{Methods}

\section{Design and sample}

This was a multi-stage stratified cluster sampling study. Firstly, according to the geographical location, economic conditions and left-behind students, in Hunan Province to select a representative of 4 townships as a survey township, and then in each township to choose a secondary school, in each secondary school of the first to the third grade were randomly selected two classes in the investigation. The inclusion criteria were as follows: (1) aged between 6 and 16 years and (2) the parents of participant have been away for more than half a year and (3) willing to participate in the study. Exclude the adolescents with cognitive impairment. Data were collected between April 13 and 17, 2020.

Typically, a median sample size of 200 is recommended for SEM analysis. An a priori sample size calculator for SEM was applied which is popular and publicly designed for calculating SEM sample sizes (https://www.danielsoper.com/statcalc/calculator.aspx?id=89). The minimum sample size with a moderate effect (0.3), at a power value of 0.95 , including three latent and 24 observed variables (all observed indicators and socio-demographic variables), and with an a of .05 was calculated as 700 . Hence, we chose a minimum sample size of 700 .

Questionnaires were distributed $(\mathrm{n}=789)$, and 751 usable responses were obtained, corresponding to a return rate of $95.18 \%$.

\section{Measurements}

\section{Socio-demographic characteristics}


Several socio-demographic characteristics such as gender, age, grade, whether the only child, household income, parents go out, the attitude of parents to go out to work and other general conditions of leftbehind junior high school students were included.

\section{Adolescent Self-rating Life Events Checklist (ASLEC)}

The Adolescent Self-rating Life Events Checklist(ASLEC) developed by Liu ${ }^{[40]}$ which was applied to measure the frequency and intensity of negative life-events in left-behind junior high school student's life. This scale includes 27 items, including 5 factors: punishment, loss, interpersonal pressure, learning pressure and adaptation. The scale adopted the 6-point Likert scale from 1 (not at all)to 6 (very much). The higher the score, the more negative events they faced. The scale reportedly has good reliability and validity with Cronbach's a was 0.95 , Split-Half Reliability was 0.88 , and Test-Retest Reliability was 0.69 . In this study, the Cronbach's a of the scale was 0.91 .

\section{Rosenberg Self-esteem Scale (RSES)}

The Rosenberg Self-Esteem Scale (RSES) was designed by Rosenberg ${ }^{[41]}$ and translated by Ji and Yu ${ }^{[42]}$, which is a more extensive assessment of self-esteem, with high authority. This scale includes 10 items, which are set on a 4-point Likert scale from 1 (strongly disagree) to 4 (strongly agree). Overall self-esteem factor can be calculated with the sum score ranging from 10 to 40 . A higher score indicates higher selfesteem. Cronbach's a was 0.78 for the Chinese version and 0.80 for this study.

\section{Resilience Scale For Chinese Adolescents}

The Resilience Scale for Chinese Adolescents was developed by Hu and Gan ${ }^{[43]}$ was applied to measure the resilience of left-behind junior high school students. This scale includes 27 items, including 5 factors: target focus, emotional control, positive cognition, family support and interpersonal assistance. It is a 5point scale ( $1-5$, representing from 'never' to 'always'), higher scores reflecting greater resilience. Cronbach's a was 0.85 for the Chinese version and 0.82 for this study.

\section{Coping Style Questionnaire (CSQ)}

The coping style ${ }^{[44]}$ was measured by the 20-item Simplified Coping Style Questionnaire (CSQ) which was developed by Xie. Responses for each question range from 0 to 3 ( $0=$ never do, $1=$ seldom do, $2=$ often do, 3 = always do). This scale includes two sub-scales to assess positive coping (item 1 to 12) and negative coping (item 13 to 20). The higher score of each dimension indicated frequent usage of this type of coping. The internal consistency measured by Cronbach's a was found to be $0.90,0.89$ and 0.78 for subscale and the whole scale respectively. In this study, the Cronbach's a of the scale was 0.80 . 


\section{Buss-warren Aggression Questionnaire (BWAQ)}

The Buss-Warren Aggression Questionnaire (BWAQ) was designed by Buss ${ }^{[45]}$ and translated by Huang [46]. The scale is a self-assessment questionnaire with 34 items, including subscales that measure aggression in five areas: Physical aggression, Anger, Verbal aggression, Indirect aggression, Hostility. The scale adopted the 5-point Likert scale from 1 (strongly disagree)to 5 (strongly agree). The higher the score, the more aggressive they were. The Cronbach's a of the total scale was 0.86 , and the Cronbach's a of each subscale ranged from 0.51 to 0.75 . In this study, the Cronbach's a was 0.920 .

\section{Ethical Considerations}

The study was reviewed by the Institutional Review Board in the researchers' university (Ethical Grant Number: E201946). Prior to the survey, the researchers informed subjects of the purpose, method, and considerations of the study and they could quit at any time during the filling process. Researchers issued the anonymous questionnaire to all eligible left-behind junior high school students after acquiring written informed consent. A quiet and private meeting room was provided for participants to complete the questionnaire. Questionnaires were retrieved immediately, and only the researchers had access to the data. The cover page of the questionnaire contained contact information of psychological consultation, should they need to.

\section{Data analysis}

Statistical analyses were conducted with IBM SPSS Statistics version20.0 and AMOS 20.0(IBM, Chicago, Illinois). Descriptive data were presented as means and standard deviations. Pearson's correlation analyses were used for correlations between two variables. Analyses of variance and independent sample t-tests were used to analyze the significance of socio-demographic differences among variable scores in the model. This helped to identify socio-demographic characteristics for inclusion as covariates in the model. The hypothesized model comprised 3 latent variables and 24 observed variables(figure 2). To verify the SEM, the incremental fit index (IFI), comparative fit index (CFI), gamma goodness-of-fit index (GFI), normed fit index (NFI), and root mean square error of approximation (RMSEA) were used as model fit indicators. Values of $\mathrm{CFI}, \mathrm{IFI}, \mathrm{NFI}$, and $\mathrm{GFI}>0.90$ are considered to reflect a good model fit. RMSEA values $<0.05$ suggest good fit, while values up to 0.08 indicate reasonable errors of approximation, indicating an acceptable fit. The parsimony of the hypothetical model was improved by dismissal of standardized path coefficients with small effects (absolute values $<0.10$ ).

\section{Results}

\section{Analysis about general data of left-behind junior high school students}


Table 1 shows there was a significant difference among aggressive behavior of junior high school students with different ages. Regarding to aggressive behavior, they have significantly differenced on gender. The different household income has tremendous influences on the left-behind junior high school students. The difference of life events of left-behind junior high school students whose fathers go out to work is significant. And the attitude of left-behind junior high school students to their parents migrant work is different, their resilience and aggressive behavior also have significant differences. 
Table 1

Relationships among socio-demographic characteristics and variable scores

\begin{tabular}{|c|c|c|c|c|c|c|c|}
\hline Variables & $N(\%)$ & Resilience & $\begin{array}{l}\text { Life } \\
\text { events }\end{array}$ & $\begin{array}{l}\text { Self- } \\
\text { esteem }\end{array}$ & $\begin{array}{l}\text { Positive } \\
\text { coping }\end{array}$ & $\begin{array}{l}\text { Negative } \\
\text { coping }\end{array}$ & $\begin{array}{l}\text { Aggressive } \\
\text { behavior }\end{array}$ \\
\hline \multicolumn{8}{|l|}{ Age, y } \\
\hline 11 & $6(0.8 \%)$ & $\begin{array}{l}87.00 \pm \\
5.80\end{array}$ & $\begin{array}{l}73.00 \\
\pm \\
20.00\end{array}$ & $\begin{array}{l}25.33 \\
\pm 3.78\end{array}$ & $\begin{array}{l}15.33 \pm \\
4.59\end{array}$ & $\begin{array}{l}6.83 \pm \\
3.31\end{array}$ & $\begin{array}{l}74.33 \pm \\
17.65\end{array}$ \\
\hline 12 & $114(15.2 \%)$ & $\begin{array}{l}93.19 \pm \\
17.87\end{array}$ & $\begin{array}{l}72.95 \\
\pm \\
25.46\end{array}$ & $\begin{array}{l}28.17 \\
\pm 5.40\end{array}$ & $\begin{array}{l}19.05 \pm \\
6.60\end{array}$ & $\begin{array}{l}8.04 \pm \\
4.86\end{array}$ & $\begin{array}{l}76.55 \pm \\
22.41\end{array}$ \\
\hline 13 & $266(35.4 \%)$ & $\begin{array}{l}96.69 \pm \\
13.31\end{array}$ & $\begin{array}{l}77.69 \\
\pm \\
22.78\end{array}$ & $\begin{array}{l}27.34 \\
\pm 5.43\end{array}$ & $\begin{array}{l}19.04 \pm \\
6.53\end{array}$ & $\begin{array}{l}8.84 \pm \\
4.56\end{array}$ & $\begin{array}{l}81.09 \pm \\
22.99\end{array}$ \\
\hline 14 & $273(36.4 \%)$ & $\begin{array}{l}91.92 \pm \\
14.70\end{array}$ & $\begin{array}{l}73.67 \\
\pm \\
22.14\end{array}$ & $\begin{array}{l}28.09 \\
\pm 4.84\end{array}$ & $\begin{array}{l}18.90 \pm \\
6.76\end{array}$ & $\begin{array}{l}8.63 \pm \\
4.67\end{array}$ & $\begin{array}{l}81.89 \pm \\
23.14\end{array}$ \\
\hline 15 & $89(11.9 \%)$ & $\begin{array}{l}87.34 \pm \\
13.01\end{array}$ & $\begin{array}{l}78.15 \\
\pm \\
26.83\end{array}$ & $\begin{array}{l}27.61 \\
\pm 4.54\end{array}$ & $\begin{array}{l}17.50 \pm \\
7.32\end{array}$ & $\begin{array}{l}8.91 \pm \\
4.52\end{array}$ & $\begin{array}{l}87.06 \pm \\
21.89\end{array}$ \\
\hline 16 & $3(0.4 \%)$ & $\begin{array}{l}92.33 \pm \\
11.68\end{array}$ & $\begin{array}{l}67.67 \\
\pm \\
23.03\end{array}$ & $\begin{array}{l}27.67 \\
\pm 4.04\end{array}$ & $\begin{array}{l}16.67 \pm \\
13.01\end{array}$ & $\begin{array}{l}13.00 \pm \\
5.20\end{array}$ & $\begin{array}{l}61.54 \pm \\
19.30 *\end{array}$ \\
\hline \multicolumn{8}{|l|}{ Gender } \\
\hline Male & $379(50.5 \%)$ & $\begin{array}{l}91.62 \pm \\
14.42\end{array}$ & $\begin{array}{l}76.22 \\
\pm \\
23.35\end{array}$ & $\begin{array}{l}27.65 \\
\pm 5.18\end{array}$ & $\begin{array}{l}18.46 \pm \\
6.56\end{array}$ & $\begin{array}{l}9.10 \pm \\
4.67\end{array}$ & $\begin{array}{l}82.62 \pm \\
22.87\end{array}$ \\
\hline Female & $372(49.5 \%)$ & $\begin{array}{l}91.25 \pm \\
15.40\end{array}$ & $\begin{array}{l}74.68 \\
\pm \\
23.65\end{array}$ & $\begin{array}{l}27.86 \\
\pm 5.01\end{array}$ & $\begin{array}{l}19.08 \pm \\
6.91\end{array}$ & $\begin{array}{l}8.19 \pm \\
4.57^{\star}\end{array}$ & $\begin{array}{l}80.03 \pm \\
23.01\end{array}$ \\
\hline \multicolumn{8}{|l|}{ Grade } \\
\hline 7 & $221(29.4 \%)$ & $\begin{array}{l}91.31 \pm \\
16.27\end{array}$ & $\begin{array}{l}77.13 \\
\pm \\
24.88\end{array}$ & $\begin{array}{l}27.38 \\
\pm 5.73\end{array}$ & $\begin{array}{l}18.46 \pm \\
6.16\end{array}$ & $\begin{array}{l}8.78 \pm \\
4.72\end{array}$ & $\begin{array}{l}78.91 \pm \\
22.36\end{array}$ \\
\hline 8 & $263(35.0 \%)$ & $\begin{array}{l}92.03 \pm \\
13.56\end{array}$ & $\begin{array}{l}74.29 \\
\pm \\
20.96\end{array}$ & $\begin{array}{l}27.71 \\
\pm 4.75\end{array}$ & $\begin{array}{l}19.00 \pm \\
7.00\end{array}$ & $\begin{array}{l}8.58 \pm \\
4.52\end{array}$ & $\begin{array}{l}80.15 \pm \\
23.32\end{array}$ \\
\hline 9 & $267(35.6 \%)$ & $\begin{array}{l}90.95 \pm \\
14.86\end{array}$ & $\begin{array}{l}75.65 \\
24.61\end{array}$ & $\begin{array}{l}28.13 \\
4.86\end{array}$ & $\begin{array}{l}18.91 \\
6.81\end{array}$ & $\begin{array}{l}8.67 \pm \\
4.65\end{array}$ & $\begin{array}{l}84.34 \pm \\
22.75\end{array}$ \\
\hline
\end{tabular}

${ }^{*} P<.05$; these scores were significantly higher or lower than those for other groups within the sociodemographic characteristic 


\begin{tabular}{|c|c|c|c|c|c|c|c|}
\hline Variables & $\mathbf{N}(\%)$ & Resilience & $\begin{array}{l}\text { Life } \\
\text { events }\end{array}$ & $\begin{array}{l}\text { Self- } \\
\text { esteem }\end{array}$ & $\begin{array}{l}\text { Positive } \\
\text { coping }\end{array}$ & $\begin{array}{l}\text { Negative } \\
\text { coping }\end{array}$ & $\begin{array}{l}\text { Aggressive } \\
\text { behavior }\end{array}$ \\
\hline \multicolumn{8}{|l|}{ The Only Child } \\
\hline Yes & $112(14.9 \%)$ & $\begin{array}{l}91.98 \pm \\
15.16\end{array}$ & $\begin{array}{l}78.49 \\
\pm \\
27.86\end{array}$ & $\begin{array}{l}27.65 \\
\pm 4.66\end{array}$ & $\begin{array}{l}18.45 \pm \\
8.15\end{array}$ & $\begin{array}{l}8.14 \pm \\
4.09\end{array}$ & $\begin{array}{l}80.40 \pm \\
23.59\end{array}$ \\
\hline No & 639(85.1\%) & $\begin{array}{l}91.32 \pm \\
14.74\end{array}$ & $\begin{array}{l}75.18 \\
\pm \\
22.70\end{array}$ & $\begin{array}{l}27.75 \\
\pm 5.17\end{array}$ & $\begin{array}{l}18.85 \pm \\
6.42\end{array}$ & $\begin{array}{l}8.74 \pm \\
4.70\end{array}$ & $\begin{array}{l}81.49 \pm \\
22.89\end{array}$ \\
\hline \multicolumn{8}{|l|}{$\begin{array}{l}\text { Household } \\
\text { Income/y }\end{array}$} \\
\hline$<10000$ & $125(16.6 \%)$ & $\begin{array}{l}91.24 \pm \\
14.46^{\star}\end{array}$ & $\begin{array}{l}78.02 \\
\pm \\
24.20\end{array}$ & $\begin{array}{l}28.50 \\
\pm 4.68\end{array}$ & $\begin{array}{l}19.90 \pm \\
6.49 *\end{array}$ & $\begin{array}{l}9.35 \pm \\
4.64\end{array}$ & $\begin{array}{l}81.55 \pm \\
24.76\end{array}$ \\
\hline $10000-20000$ & $122(16.2 \%)$ & $\begin{array}{l}95.64 \pm \\
16.53\end{array}$ & $\begin{array}{l}73.61 \\
\pm \\
24.75\end{array}$ & $\begin{array}{l}28.03 \\
\pm 6.36\end{array}$ & $\begin{array}{l}20.80 \pm \\
6.89\end{array}$ & $\begin{array}{l}8.27 \pm \\
4.99\end{array}$ & $\begin{array}{l}76.95 \pm \\
22.68\end{array}$ \\
\hline$>20000$ & $116(15.4 \%)$ & $\begin{array}{l}93.62 \pm \\
14.89\end{array}$ & $\begin{array}{l}75.89 \\
\pm \\
22.17\end{array}$ & $\begin{array}{l}27.85 \\
\pm 4.58\end{array}$ & $\begin{array}{l}19.32 \pm \\
7.31\end{array}$ & $\begin{array}{l}9.80 \pm \\
4.96\end{array}$ & $\begin{array}{l}82.12 \pm \\
24.23\end{array}$ \\
\hline Unclear & $388(51.7 \%)$ & $\begin{array}{l}89.60 \pm \\
14.13\end{array}$ & $\begin{array}{l}75.28 \\
\pm \\
23.23\end{array}$ & $\begin{array}{l}27.34 \\
\pm 4.89\end{array}$ & $\begin{array}{l}17.66 \pm \\
6.32\end{array}$ & $\begin{array}{l}8.64 \pm \\
4.45\end{array}$ & $\begin{array}{l}82.15 \pm \\
22.01\end{array}$ \\
\hline \multicolumn{8}{|l|}{$\begin{array}{l}\text { Father out for } \\
\text { work }\end{array}$} \\
\hline Yes & $\begin{array}{l}684 \\
(91.1 \%)\end{array}$ & $\begin{array}{l}91.38 \pm \\
14.93\end{array}$ & $\begin{array}{l}76.52 \\
\pm \\
23.48^{*}\end{array}$ & $\begin{array}{l}27.72 \\
\pm 5.12\end{array}$ & $\begin{array}{l}18.75 \pm \\
6.65\end{array}$ & $\begin{array}{l}8.65 \pm \\
4.66\end{array}$ & $\begin{array}{l}81.40 \pm \\
22.84\end{array}$ \\
\hline No & 67 (8.9\%) & $\begin{array}{l}91.04 \pm \\
12.93\end{array}$ & $\begin{array}{l}68.79 \\
\pm \\
20.66\end{array}$ & $\begin{array}{l}27.90 \\
\pm 4.86\end{array}$ & $\begin{array}{l}19.14 \pm \\
7.03\end{array}$ & $\begin{array}{l}9.25 \pm \\
4.19\end{array}$ & $\begin{array}{l}82.72 \pm \\
23.43\end{array}$ \\
\hline \multicolumn{8}{|l|}{$\begin{array}{l}\text { Mother out for } \\
\text { work }\end{array}$} \\
\hline Yes & $\begin{array}{l}474 \\
(63.1 \%)\end{array}$ & $\begin{array}{l}91.03 \pm \\
14.08\end{array}$ & $\begin{array}{l}75.81 \\
\pm \\
22.99\end{array}$ & $\begin{array}{l}27.65 \\
\pm 4.91\end{array}$ & $\begin{array}{l}18.51 \pm \\
6.64\end{array}$ & $\begin{array}{l}8.86 \pm \\
4.65\end{array}$ & $\begin{array}{l}81.22 \pm \\
22.42\end{array}$ \\
\hline No & $277(36.9 \%)$ & $\begin{array}{l}92.21 \pm \\
15.70\end{array}$ & $\begin{array}{l}75.55 \\
\pm \\
24.31\end{array}$ & $\begin{array}{l}27.89 \\
\pm 5.38\end{array}$ & $\begin{array}{l}19.24 \pm \\
6.65\end{array}$ & $\begin{array}{l}8.36 \pm \\
4.58\end{array}$ & $\begin{array}{l}81.19 \pm \\
24.08\end{array}$ \\
\hline
\end{tabular}

$\star P<.05$; these scores were significantly higher or lower than those for other groups within the sociodemographic characteristic 


\begin{tabular}{|c|c|c|c|c|c|c|c|}
\hline Variables & $\mathbf{N}(\%)$ & Resilience & $\begin{array}{l}\text { Life } \\
\text { events }\end{array}$ & $\begin{array}{l}\text { Self- } \\
\text { esteem }\end{array}$ & $\begin{array}{l}\text { Positive } \\
\text { coping }\end{array}$ & $\begin{array}{l}\text { Negative } \\
\text { coping }\end{array}$ & $\begin{array}{l}\text { Aggressive } \\
\text { behavior }\end{array}$ \\
\hline \multicolumn{8}{|l|}{$\begin{array}{l}\text { The attitude } \\
\text { of parents to } \\
\text { go out to work }\end{array}$} \\
\hline Strongly agree & $91(12.1 \%)$ & $\begin{array}{l}94.50 \pm \\
15.49^{\star}\end{array}$ & $\begin{array}{l}71.53 \\
\pm \\
24.08\end{array}$ & $\begin{array}{l}28.38 \\
\pm 5.93\end{array}$ & $\begin{array}{l}19.81 \pm \\
7.04\end{array}$ & $\begin{array}{l}8.69 \pm \\
5.02\end{array}$ & $\begin{array}{l}75.58 \pm \\
24.39\end{array}$ \\
\hline Agree & $426(56.7 \%)$ & $\begin{array}{l}92.07 \pm \\
14.30\end{array}$ & $\begin{array}{l}75.74 \\
\pm \\
23.07\end{array}$ & $\begin{array}{l}27.93 \\
\pm 4.80\end{array}$ & $\begin{array}{l}18.73 \pm \\
6.49\end{array}$ & $\begin{array}{l}8.64 \pm \\
4.49\end{array}$ & $\begin{array}{l}80.12 \pm \\
21.75\end{array}$ \\
\hline Indifferent & $104(13.8 \%)$ & $\begin{array}{l}87.80 \pm \\
15.67\end{array}$ & $\begin{array}{l}76.39 \\
\pm \\
23.40\end{array}$ & $\begin{array}{l}26.77 \\
\pm 5.71\end{array}$ & $\begin{array}{l}17.50 \pm \\
6.49\end{array}$ & $\begin{array}{l}9.22 \pm \\
4.78\end{array}$ & $\begin{array}{l}87.40 \pm \\
22.58^{\star}\end{array}$ \\
\hline Disagree & $105(14.0 \%)$ & $\begin{array}{l}89.88 \pm \\
15.31\end{array}$ & $\begin{array}{l}76.48 \\
\pm \\
22.38\end{array}$ & $\begin{array}{l}27.42 \\
\pm 4.70\end{array}$ & $\begin{array}{l}19.16 \pm \\
6.76\end{array}$ & $\begin{array}{l}8.82 \pm \\
4.62\end{array}$ & $\begin{array}{l}86.51 \pm \\
24.61\end{array}$ \\
\hline $\begin{array}{l}\text { Strongly } \\
\text { disagree }\end{array}$ & $25(3.3 \%)$ & $\begin{array}{l}86.91 \pm \\
11.44\end{array}$ & $\begin{array}{l}81.00 \\
\pm \\
31.37\end{array}$ & $\begin{array}{l}27.40 \\
\pm 5.56\end{array}$ & $\begin{array}{l}19.01 \pm \\
7.78\end{array}$ & $\begin{array}{l}8.12 \\
\pm .75\end{array}$ & $\begin{array}{l}82.32 \pm \\
22.60\end{array}$ \\
\hline
\end{tabular}

Descriptive analysis about the aggressive behavior of left-behind junior high school students and its influencing factors

Table 2 shows the descriptive analysis of resilience, life events, self-esteem, coping styles and aggressive behaviors of left-behind junior high school students. The average score of resilience was $(91.41 \pm 14.85)$, life events was $(75.58 \pm 23.47)$, self-esteem was $(27.76 \pm 5.10)$, positive coping was (18.78 \pm 6.71$)$, negative coping was $(8.66 \pm 4.62)$, aggressive behavior was $(81.28 \pm 22.91)$.

Table 2

Descriptive Statistics of the Measured Variables $(n=751)$

\begin{tabular}{|lllll|}
\hline Variables & Mean & SD & Median & Range \\
\hline Resilience & 91.41 & 14.85 & 89.00 & $50.00-131.00$ \\
\hline Life events & 75.58 & 23.47 & 73.00 & $28.00-147.00$ \\
\hline Self-esteem & 27.76 & 5.10 & 28.00 & $10.00-40.00$ \\
\hline Positive coping & 18.78 & 6.71 & 19.00 & $0.00-36.00$ \\
\hline Negative coping & 8.66 & 4.62 & 8.00 & $0.00-22.00$ \\
\hline Aggressive behavior & 81.28 & 22.91 & 82.00 & $37.00-152.00$ \\
\hline
\end{tabular}


The relationship between aggressive behavior and its influencing factors of left-behind junior high school students

Table 3 shows the relationship among resilience, life events, self-esteem, coping styles and aggressive behaviors of left-behind junior high school students. Resilience of left-behind junior high school students is negatively correlated with life events, negative coping, aggressive behaviors, but positively correlated with self-esteem, positive coping. Life events have negative associations with self-esteem, have positive associations with negative coping and aggressive behaviors, have no associations with positive coping. Self-esteem can positively predict the positive coping, negatively predict the negative coping and aggressive behavior. Positive coping positively affects the negative coping, but cannot affect the aggressive behavior. Negative coping is positively correlated with aggressive behavior.

Table 3

Correlations Among the Measured Variables $(n=751)$

\begin{tabular}{|c|c|c|c|c|c|c|}
\hline Variables & Resilience & $\begin{array}{l}\text { Life } \\
\text { events }\end{array}$ & $\begin{array}{l}\text { Self- } \\
\text { esteem }\end{array}$ & $\begin{array}{l}\text { Positive } \\
\text { coping }\end{array}$ & $\begin{array}{l}\text { Negative } \\
\text { coping }\end{array}$ & $\begin{array}{l}\text { Aggressive } \\
\text { behavior }\end{array}$ \\
\hline Resilience & 1 & & & & & \\
\hline Life events & $-0.363^{a}$ & 1 & & & & \\
\hline Self-esteem & $0.624^{a}$ & $-0.373^{a}$ & 1 & & & \\
\hline Positive coping & $0.498^{a}$ & $-0.025^{b}$ & $0.430^{\mathrm{a}}$ & 1 & & \\
\hline $\begin{array}{l}\text { Negative } \\
\text { coping }\end{array}$ & $-0.255^{a}$ & $0.283^{a}$ & $-0.174^{a}$ & $0.228^{a}$ & 1 & \\
\hline $\begin{array}{l}\text { Aggressive } \\
\text { behavior }\end{array}$ & $-0.450^{a}$ & $0.416^{a}$ & $-0.348^{a}$ & $-0.028^{b}$ & $0.429 a$ & 1 \\
\hline \multicolumn{7}{|l|}{ a $P=.000$} \\
\hline $\mathrm{b} P>0.05$ & & & & & & \\
\hline
\end{tabular}

\section{Structural Equation Modeling}

The structural equation model was initially tested, and after removing pathways with vary small effects (absolute values $<0.10$ ), a modified model with good model fit was obtained (Fig. 3 ). The parameters of the model were as follows: $\chi 2=687.50, \mathrm{DF}=150, \mathrm{X} 2 / \mathrm{DF}=4.583<5, \mathrm{GFI}=0.910>0.9, \mathrm{NFI}=0.903>0.9, \mathrm{IFI}$ $=0.923>0.9, \mathrm{CFI}=0.922>0.9, \mathrm{RMSEA}=0.069<0.8$ (Table 4). Although $\mathrm{P}<0.05$, all other measurement models were determined to have good fit indices, and the sample size was relatively large. Therefore, this model was acceptable ${ }^{[47]}$. The standardized parameters of the final model are presented in Fig. 3. Table 5 and Table 6 show the direct effect, indirect effect and total effect among the relevant factors. 
Table 4

Comparison of Model Fit for the Modified Model to the Hypothetical Model

\begin{tabular}{|lllllllll|}
\hline Model & $\chi \mathbf{2}(\boldsymbol{P})$ & $\boldsymbol{d f}$ & $\boldsymbol{\chi 2} \mathbf{d f}$ & GFI & NFI & IFI & CFI & RMSEA \\
\hline Reference & $>.05$ & & $<5$ & $0.9-1$ & $0.9-1$ & $0.9-1$ & $0.9-1$ & $<0.08$ \\
\hline Hypothetical & $1469.99(.00)$ & 239 & 6.151 & 0.842 & 0.803 & 0.830 & 0.829 & 0.083 \\
\hline Modified & $687.50(.00)$ & 150 & 4.583 & 0.910 & 0.903 & 0.923 & 0.922 & 0.069 \\
\hline $\begin{array}{l}\text { Abbreviations: GFI, goodness-of-fit index; NFI, normed fit index; IFI, Incremental Fit Index; CFI, } \\
\text { comparative of fit index; } d f \text {, degree of freedom; RMSEA, root mean square error of approximation; }\end{array}$ \\
\hline
\end{tabular}


Table 5

Standardized Direct, Indirect, and Total Effects for the Modified Model

\begin{tabular}{|c|c|c|c|c|}
\hline $\begin{array}{l}\text { Endogenous } \\
\text { Variables }\end{array}$ & $\begin{array}{l}\text { Exogenous } \\
\text { Variables }\end{array}$ & $\begin{array}{l}\text { Standardized Direct } \\
\text { Effects }\end{array}$ & $\begin{array}{l}\text { Standardized } \\
\text { Indirect } \\
\text { Effects }\end{array}$ & $\begin{array}{l}\text { Standardized Total } \\
\text { Effects }\end{array}$ \\
\hline \multirow{6}{*}{$\begin{array}{l}\text { Aggressive } \\
\text { behavior }\end{array}$} & Resilience & -0.626 & & -0.626 \\
\hline & Positive coping & 0.256 & -0.275 & -0.019 \\
\hline & Negative coping & 0.148 & 0.165 & 0.313 \\
\hline & Life events & 0.147 & 0.361 & 0.508 \\
\hline & $\begin{array}{l}\text { household } \\
\text { income }\end{array}$ & & 0.002 & 0.002 \\
\hline & Self-esteem & & -0.253 & -0.253 \\
\hline \multirow[t]{5}{*}{ Resilience } & Positive coping & 0.439 & & 0.439 \\
\hline & Life events & -0.263 & -0.287 & -0.550 \\
\hline & Negative coping & -0.273 & 0.135 & -0.138 \\
\hline & Self-esteem & 0.391 & 0.208 & 0.599 \\
\hline & $\begin{array}{l}\text { household } \\
\text { income }\end{array}$ & & -0.050 & -0050 \\
\hline \multirow[t]{4}{*}{ Positive coping } & Self-esteem & 0.473 & & 0.473 \\
\hline & Negative coping & 0.307 & & 0.307 \\
\hline & $\begin{array}{l}\text { household } \\
\text { income }\end{array}$ & -0.113 & & -0.113 \\
\hline & Life events & & -0.104 & -0.104 \\
\hline $\begin{array}{l}\text { Negative } \\
\text { coping }\end{array}$ & Life events & 0.294 & & 0.294 \\
\hline Self-esteem & Life events & -0.411 & & -0.411 \\
\hline
\end{tabular}


Table 6

Maximum likelihood estimates of the modified model.

\begin{tabular}{|c|c|c|c|c|c|c|}
\hline Pathway & & $\begin{array}{l}\text { Non- } \\
\text { standardized } \\
\text { Coefficients }\end{array}$ & $\begin{array}{l}\text { Standardized } \\
\text { Coefficients }\end{array}$ & $\begin{array}{l}\text { Standard } \\
\text { Errors }\end{array}$ & $\begin{array}{l}\text { Critical } \\
\text { Ratio }\end{array}$ & $P$ \\
\hline Self-esteem & Life events & -0.594 & -0.411 & 0.055 & -10.761 & 0.000 \\
\hline $\begin{array}{l}\text { Negative } \\
\text { coping }\end{array}$ & Life events & 0.385 & 0.294 & 0.050 & 7.655 & 0.000 \\
\hline $\begin{array}{l}\text { Positive } \\
\text { coping }\end{array}$ & $\begin{array}{l}\text { Negative } \\
\text { coping }\end{array}$ & 0.446 & 0.307 & 0.045 & 9.932 & 0.000 \\
\hline $\begin{array}{l}\text { Positive } \\
\text { coping }\end{array}$ & $\begin{array}{l}\text { Self- } \\
\text { esteem }\end{array}$ & 0.624 & 0.473 & 0.041 & 15.319 & 0.000 \\
\hline $\begin{array}{l}\text { Positive } \\
\text { coping }\end{array}$ & $\begin{array}{l}\text { household } \\
\text { income }\end{array}$ & -0.657 & -0.113 & 0.178 & -3.700 & 0.000 \\
\hline Resilience & $\begin{array}{l}\text { Positive } \\
\text { coping }\end{array}$ & 0.157 & 0.439 & 0.015 & 10.560 & 0.000 \\
\hline Resilience & $\begin{array}{l}\text { Negative } \\
\text { coping }\end{array}$ & -0.142 & -0.273 & 0.019 & -7.622 & 0.000 \\
\hline Resilience & $\begin{array}{l}\text { Self- } \\
\text { esteem }\end{array}$ & 0.184 & 0.391 & 0.019 & 9.522 & 0.000 \\
\hline Resilience & Life events & -0.179 & -0.263 & 0.027 & -6.645 & 0.000 \\
\hline $\begin{array}{l}\text { Aggressive } \\
\text { behavior }\end{array}$ & Resilience & -1.314 & -0.626 & 0.184 & -7.131 & 0.000 \\
\hline $\begin{array}{l}\text { Aggressive } \\
\text { behavior }\end{array}$ & $\begin{array}{l}\text { Positive } \\
\text { coping }\end{array}$ & 0.192 & 0.256 & 0.045 & 4.307 & 0.000 \\
\hline $\begin{array}{l}\text { Aggressive } \\
\text { behavior }\end{array}$ & $\begin{array}{l}\text { Negative } \\
\text { coping }\end{array}$ & 0.162 & 0.148 & 0.048 & 3.385 & 0.000 \\
\hline $\begin{array}{l}\text { Aggressive } \\
\text { behavior }\end{array}$ & Life events & 0.211 & 0.147 & 0.071 & 2.966 & 0.003 \\
\hline
\end{tabular}

Life events (effect value $=0.508)$, mental resilience $(-0.626)$, positive coping $(-0.019)$, negative coping (0.313), self-esteem ( -0.253$)$ and household income $(0.002)$ have different degrees of impact on the aggressive behavior of left-behind junior high school students. Therefore, $\mathrm{H} 1$ was supported and clarified.

Life events showed a significant effect on aggressive behavior (0.508), the majority of which was indirect effect (0.361) mainly via self-esteem (0.104), mental resilience (0.165) and negative coping (0.092), and positive coping played a certain intermediary effect when self-esteem(-0.009) and negative coping $(-0.005)$ produced aggressive behavior. Therefore, $\mathrm{H} 2$ was supported and clarified. 
Positive coping has direct (0.256) and indirect (-0.275) effects on aggressive behavior (-0.019), in which the indirect effect is generated by resilience $(-0.275)$. Therefore, $\mathrm{H} 3 \mathrm{a}$ was supported and clarified. Negative coping has direct (0.148) and indirect (0.165) effects on aggressive behavior (0.313), among which the indirect effects are generated by resilience (0.171) and positive coping (-0.006). Therefore, H3b was supported and clarified. Self-esteem has direct (0.391) and indirect $(0.208)$ effects on mental resilience (0.599), among which the indirect effects are generated by positive coping $(0.208)$. Therefore, $\mathrm{H} 3 \mathrm{C}$ was partially supported and clarified. Negative coping has direct $(-0.273)$ and indirect $(0.135)$ effects on mental resilience $(-0.138)$, in which the indirect effect is generated by positive coping $(0.135)$. Therefore, H3d was supported and clarified.

In general, Life events, self-esteem, resilience, positive coping, negative coping and household income have different direct or indirect effects on the aggressive behavior of left-behind junior high school students. Among them, self-esteem, resilience, positive coping and negative coping are the important mediating factors between life events and aggressive behavior of left-behind junior high school students.

\section{Discussion}

This study detected and clarified that socio-demographic characteristics, life events, self-esteem, resilience and coping style have a direct or indirect influence on the aggressive behavior of left-behind junior high school students, and further analyzes the specific effect path of the influencing factors on the aggressive behavior of the left-behind junior high school students through the structural equation model, which provides the evidence for helping parents, teachers and temporary guardians to implement targeted interventions to effectively prevent and mitigate the aggressive behavior of left-behind junior high school students.

\section{Analysis on the status and influencing factors of left-behind junior high school students' aggressive behavior}

The results of this study show that the aggressive behavior score of left-behind junior high school students is (81.28 \pm 22.91$)$, which is at a medium level. It indicates that left-behind junior high school students have aggressive behaviors, which are mainly affected by their age and their attitude towards their parents' migrant work. This may be related to the rapid development of individual personality and their different attachment degrees to their parents in adolescence. Romero ${ }^{[48]}$ found that the negative emotions and aggressive behaviors of individuals in the adolescence increase by age. Yao's study ${ }^{[49]}$ of aggressive behavior among left-behind children found that the higher the parent-child attachment of children in the same age group, the less the aggressive behavior. Therefore, we should pay more attention to the emotional changes of children with high possibility of aggressive behaviors, conduct timely psychological counseling, and avoid the occurrence of aggressive behaviors.

Correlation analysis found that left-behind junior high school students' aggressive behavior was significantly positively correlated with negative events and negative coping, which was consistent with 
the previous research ${ }^{[50]}$. This may be related to the adverse effects of negative events on adolescent mood and behavior as well as negative cognition ${ }^{[51]}$. Left-behind junior high school students whose fathers left home for work are significantly more affected by negative events than other groups, which may be related to the influence of absent fathers on the character of left-behind children. Feng ${ }^{[52]}$ found that the absence of a father would reduce the social adaptability and anti-frustration ability of left-behind children, making them more vulnerable to negative events. In relation to the choice of response methods, the difference of the negative and positive response score of the left-behind junior high school students with different genders and household income was statistically significant. This may be related to the

difference in psychological maturity between adolescent men and women ${ }^{[53]}$ and the difference in living environment with different household incomes ${ }^{[54]}$.

In addition, the aggressive behavior of left-behind junior high school students was negatively correlated with resilience and self-esteem, consistent with the previous study ${ }^{[55]}$. It has been proved that left-behind junior high school students with higher resilience and self-esteem will produce fewer hostile emotions and aggressive behaviors when face the stress or negative events. The resilience level of left-behind junior high school students is related to their attitude towards their parents' migrant work, which is consistent with the research results of Zhang ${ }^{[56]}$. Therefore, parents, teachers and temporary guardians can increase left-behind junior high school student's understanding of parents' migrant work by communicating and explaining with them, so as to enhance their resilience and reduce their hostile emotions and aggressive behaviors.

\section{The structural equation model of aggressive behavior of left-behind junior high school students}

According to the structural equation model, negative life events, have great influence on the aggressive behavior of left-behind junior high school students (0.508). With the increase of negative life events, the aggressive behavior of left-behind junior high school students also increases, consistent with the research results of Yang ${ }^{[57]}$. Middle school is a critical period for the physical and mental development of teenagers. Research found that ${ }^{[58]}$ the absence of parents at this stage will cause left-behind children to suffer more negative events in interpersonal communication, study, living environment and other aspects. Due to the immaturity of thought and coping style, as well as the lack of proper guidance from parents, compared with non-left-behind junior high school students, left-behind junior high school students are more likely to have violent behaviors stimulated by negative life events ${ }^{[58]}$. Therefore, reducing the impact of negative life events on its appearance should come to the first place before downgrading or preventing the occurrence of left-behind junior high school student's aggressive behavior.

Our results showed that the impact of life events on the left-behind junior high school student's aggressive behavior mainly comes from the indirect effects $(0.361)$ of resilience $(0.165)$, self-esteem (0.104) and negative coping (0.092), apart from the direct impact (0.147). Moreover, resilience $(-0.626)$, self-esteem (-0.253), negative coping (0.313), and positive coping $(-0.019)$ can also exert a certain influence on the aggressive behaviors of left-behind junior high school students through direct or indirect 
paths. Thereinto, mental resilience has the greatest impact on the aggressive behavior of left-behind junior high school students, and only has a direct negative effect, which is consistent with previous research ${ }^{[59]}$. This may be related to high resilience that can effectively mediate an individual's perception of negative information, making it easier to perceive the positive side of things and thus adapt well to a frustrating environment ${ }^{[60]}$. Under the same setback event, left-behind junior high school students with high self-esteem, psychological resilience and positive coping tendency are less likely to have aggressive behaviors, while left-behind junior high school students with high negative coping tendency are more

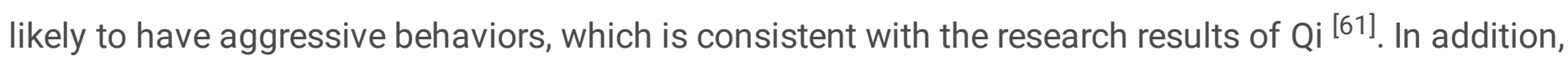
relevant studies found that ${ }^{[62-64]}$ the left-behind children's self-esteem, mental resilience and coping tendency were mainly related to their self-cognition level and psychological support from family, school and society. Therefore, implementing effective psychological intervention measures, strengthening the positive psychological support for left-behind children, and establishing a correct cognitive view of frustration can effectively improve the left-behind junior high school student's self-esteem, mental resilience and positive coping tendency, reduce the impact of negative life events on them, and prevent the occurrence of aggressive behaviors. In addition, our study found that household income can also have weak indirect positive effect on the aggressive behavior of left-behind high school students (0.002). Hence, parents, teachers or temporary guardians should pay more attention to the emotional changes of such left-behind junior high school students, and implement effective psychological interventions in a timely manner to minimize or prevent the occurrence of their aggressive behavior.

\section{Limitations}

First, participants in the study were left-behind junior high school students, hence, the applicability of this the finding is uncertain for the rest of the left behind school children. Second, because of the crosssectional design, causal relationships among the variables were constructed based on theoretical analysis and literature review, and should be interpreted with caution. Third, we only investigated the impact of life events and internal psychological traits on the left-behind junior high school student's aggressive behavior, and did not investigate or analyze the external social support of left-behind junior high school students. Future research could apply a longitudinal design to test more variables of influencing the aggressive behavior of left-behind children at different time points, so as to help caregivers effectively reduce the occurrence of left-behind children's aggressive behavior.

\section{Conclusions}

This study detected and clarified that the left-behind junior high school student's aggressive behavior was influenced by general information, life events, self-esteem, mental resilience, positive coping and negative coping. Among them, resilience, self-esteem, positive coping and negative coping play an important mediating role in the life events and aggressive behaviors of left-behind junior high school students. The left-behind junior high school students with high resilience, self-esteem and positive coping tendency are less likely to have aggressive behaviors, while left-behind junior high school students with high negative 
coping tendency are more likely to have aggressive behaviors. Moreover, high mental resilience and selfesteem left-behind children are more inclined to adopt positive coping style. The left-behind junior high school students' mental resilience is significantly positively correlated with self-esteem.

Therefore, parents, teachers and temporary guardians in the care of left-behind children should not only try to avoid possible adverse effects that the life events have on the children, but also should put emphasis on the development of left-behind children's self-awareness and evaluation ability, strengthen psychological support and active guidance of left-behind children, so as to help left-behind children to fully and objectively understand themselves. Increase their optimism about life and stress, and improve their self-esteem, psychological resilience and positive coping tendencies, thereby reducing or preventing aggressive behaviors of left-behind children and promoting their physical and mental health growth.

\section{Abbreviations}

CPT

cognitive-phenomenological-transactional theoretical

ASLEC

Adolescent Self-rating Life Events Checklist

RSES

Rosenberg Self-Esteem Scale

CSQ

Coping Style Questionnaire

BWAQ

Buss-Warren Aggression Questionnaire

\section{Declarations}

\section{Ethics approval and consent to participate}

This study was approved by Central South university's Institutional Review Board (IRB) [No: E201946] before data collection.

\section{Consent for publication}

Not applicable.

\section{Availability of data and materials}

The datasets used and/or analyzed during the current study are available from the corresponding author on reasonable request.

Competing interests 
The authors declare that they have no competing interests.

\section{Funding}

Not applicable.

\section{Authors' contributions}

JL, YPC, SLY, YXT, BYY collected and analyzed the data, JPZ and TM directed the study, Yi-Fei Li and Jie Zhang collected and analyzed the data, and were the major contributor in writing the manuscript. All authors read and approved the final manuscript.

Yi-Fei Li and Jie Zhang contributed equally to this study. They are shared the first authorship equally.

\section{Acknowledgments}

The authors wish to thank the research all the participants, the statistician, and health professionals for making this research possible.

\section{References}

[1] Yang TL, Zhu JQ, Guan HY, et al. Vision health among left-behind, non-left-behind and migrant primary school children: a comparative study. Chinese Journal of Public Health.2019, Doi:10.11847/zgggws1126174.

[2] National Bureau of statistics of the people's Republic of China, 2018 migrant workers Monitoring Survey Report [Online].2019. Available:

http://www.stats.gov.cn/tjsj/zxfb/201904/t20190429_1662268.html. [Accessed 29/4 2019].

[3] Hao W, Wu CX, Yu YZ. Aggressive behaviors among left-behind and non-left-behind children in rural China: a comparative analysis. Chinese Journal of Public Health.2019, Doi: 10.11847/zgggws1120384

[4] Jiang Y. A study on the situation of left behind children in China. Population and Family Planning.2016(06):21-22.

[5] Wang CY. The influence of ego-identity status and staying left or not on the aggressiveness of junior middle school students in frustration situation. Hebei Normal University.2019.

[6] Ministry of Education of the People's Republic of China, Number of children with emigration in China's compulsory education stage 2013-2017 [Online].2018. Available: https://www.sohu.com/a/258196622_100291829. [Accessed 8/10 2018].

[7] White Papers of the Chinese Government. 2019 White Paper on the Mental State of Left-behind Children in China [Online].2020. Available: https://baijiahao.baidu.com/s? id $=1655697134660346688 \& \mathrm{wfr}=$ spider\&for=pc. [Accessed 1/14 2020]. 
[8] Chen J. Study on psychological health and family factors in three medical university students. Southern Medical University.2017.

[9] Cheng J, Sun YH. Depression and anxiety among left-behind children in China: a systematic review. Child: care, Health and Development.2015,41(4): 515-523.

[10] Guo J, Ren XZ, Wang XH, et al. Depression among Migrant and Left-Behind Children in China in Relation to the Quality of Parent-Child and Teacher-Child Relationships. PloS one.2015,10(12). Doi: 10.1371/journal.pone.0145606.

[11] Dai Q, Chu RX. Anxiety, happiness and self-esteem of western Chinese left-behind children. Child Abuse \& Neglect.2018,86:403-413.

[12] Dong ST, Li M. The monitoring problem and the empirical study of crime in rural left-behind children. Journal of Chinese People's Public Security University (Social Sciences Edition). 2010,26(03):133-139.

[13] Zhang L, Cai C, Wang Z. Adolescent-to-Mother Psychological Aggression: The Role of Father Violence and Maternal Parenting Style. Child Abuse \& Neglect [Online].2019,98. https://doi.org/10.1016/j.chiabu.2019.104229.

[14] Zhang XL. Setback Situation for Different Self-acceptance Levels of Left-behind Junior Middle School Students' Influence on Aggression. Hebei Normal University.2017.

[15] Huang J, Tang J, Tang L, et al. Aggression and related stressful life events among Chinese adolescents living in rural areas: A cross-sectional study. Journal of affective disorders,2017,211:20-26.

[16] Paul R. Smokowski, Katie L. Cotter, Caroline I. B. Robertson, et al. Anxiety and aggression in rural youth: baseline results from the rural adaptation project. Child psychiatry and human development,2013,44:479-492.

[17] Huesmann, L. R., Eron, L. D., Lefkowitz, M. M., \& Walder, L. O. (1984). Stability of aggression over time and generations. Developmental Psychology, 20(6), 1120-1134. https://doi.org/10.1037/00121649.20.6.1120.

[18] TANG Jie, WU Yu, YU Yizhen. Structural Equation on Influencing Factors of Aggressive Behavior among Middle School Student in City. Chinese Journal of Social Medicine. 2014,31(03),193-195.

[19] Tian Wei. The Experimental Research on the Influence of Empathy Training to Left-behind Children's Aggressive Behavior. Southwestern University.2009.

[20] Ding Yan. A Study on Mental stress and Coping Style among the Left-behind Junior Middle School Students. Hunan Normal University.2012. 
[21] Wu CX. A comparative analysis of aggression in left-behind children and non-left-behind children in rural China. Huazhong University of Science and Technology.2018.

[22] Wei YH, Tang SQ. Several major stress theory models and their evaluation. PSYCHOLOGICAL SCIENCE.1998.21(5):441-444.

[23] Folkman, S., Lazarus, R. S., Dunkel-Schetter, C., DeLongis, A. \& Gruen, R. J.(1986). Dynamics of a stressful encounter: cognitive appraisal, coping, and encounter outcomes. Journal of Personality and Social Psychology, 50, 992.

[24] Miller N E, Sears R R, Mowrer O H, Doob L W, Dollard J. The frustration-aggression hypothesis. Psychological Review.1941, 48(4):337-342.

[25] Tara J. Cheetham-Blake,Hannah E. Family,Julie M. Turner-Cobb. 'Every day I worry about something': A qualitative exploration of children's experiences of stress and coping. British Journal of Health Psychology,2019,24(4): 931-952.

[26] Liu BQ. Investigation and analysis of life stress events for rural left-behind junior high school students. Education Exploration.2011(12):108-109.

[27] Li ZX. The Relationship among Adolescents' Life events, Personality Traits and Aggressive Behavior. Guangzhou University.2010.

[28] Yu QY. Study on the frustration education of college students. Chongqing Normal University.2014.

[29] Berkowitz, L. (1989). Frustration-aggression hypothesis: Examination and reformulation.

Psychological Bulletin, 106(1), 59-73. https://doi.org/10.1037/0033-2909.106.1.59.

[30] Zhang Yan. A qualitative study on adolescent's response model of replacing aggressive frustration. Nanjing Normal University.2014.

[31] Fan Xin. Research on adolescent aggressive investigation and psychological intervention. Shanxi Medical University.2013.

[32] Lin YC, Latner JD, Fung XCC. Poor Health and Experiences of Being Bullied in Adolescents: SelfPerceived Overweight and Frustration with Appearance Matter. Obesity (Silver Spring). 2018,26(2):397404.

[33] Amad S, Gray NS, Snowden RJ. Self-Esteem, Narcissism, and Aggression: Different Types of SelfEsteem Predict Different Types of Aggression. Journal of Interpersonal Violence [Online]. 2020:886260520905540. Doi: 10.1177/0886260520905540.

[34] Luthar SS, Cicchetti D, Becker B. The construct of resilience: a critical evaluation and guidelines for future work. Child Development. 2000,71(3):543-62. 
[35] Wu HC. A Study on the Relationship between Personality Trait and Resilience of High School Students with Left-behind Experience. Hebei Normal University.2014.

[36] Gao Shu. The Characteristics of the Cognitive Bias of Junior Middle School

Students of Emotional Elasticity. Shanxi Normal University.2017.

[37] Ertekin Pinar S, Yildirim G, Sayin N. Investigating the psychological resilience, self-confidence and problem-solving skills of midwife candidates. Nurse Education Today. 2018,64:144-149.

[38] Zhang XQ, Qiu Fen. The Influence of Physical Exercise on Aggressiveness of Adolescents --Chain Mediation of Coping Style and Psychological Elasticity. Journal of Harbin Sport University. 2019,37(05),85-91.

[39] Wang XY, Zhang TT. Study on the Relationship between Attack, Self-respect and Replying Modes of Higher Vocational School Student. Vocational and Technical Education. 2011. 32(20): 89-92.

[40] Liu XC, Liu LQ, Yang J, et al. The reliability validity test of the adolescent life event scale. CHINESE JOURNAL OF CLINICAL PSYCHOLOGY. 1997(01):39-41.

[41] Rosenberg, M. (1965). Society and the adolescent self-image. Princeton, NJ: Princeton University Press.

[42] Ji YF, Yu Xin, The Self-Esteem scale, SES. Chinese Mental Health Journal.1993:251-252.

[43] Hu YQ, Gan YQ. Development and Psychometric Validity of the Resilience Scale for Chinese Adolescents. Acta Psychologica Sinica.2008(08):902-912.

[44] Xie YN, Preliminary study on the reliability and validity of the simple response scale. Chinese Journal of Clinical Psychology. 1998(02):53-54.

[45] Fred B. Bryant, Bruce D. Smith. Refining the architecture of aggression: A measurement model for the buss-perry aggression questionnaire. Journal of Research in Personality:2011, 35(2):138-167.

[46] Huang Juan. Development and Application of Buss-Warren Aggression Questionnaire and its Norm among Rural Students Age 9 to 18 in China. Huazhong University of science and technology.2017.

[47] Wu M L. Structural Equation Modeling: the application and operation of AMOS. Chongqing: Chongqing University Press; 2009.

[48] Romero E, Alonso C. Maladaptative personality traits in adolescence: behavioural, emotional and motivational correlates of the PID-5-BF scales. Psicothema. 2019.31(3):263-270.

[49] Yao RY, Liu Feng, Zhuang WR, et al. The aggressive behavior and parental attachment in left-behind and non-left-behind senior pupils in north of Anhui Province and their relationships. Journal of Bengbu 
Medical College.2015,40(05):650-655.

[50] Jing LS, Feng Jun, Xu Tao. The Correlation between Adolescents' Aggression and Stressful Event or Coping Style. China Journal of Health Psychology. 2014,22(01):53-55.

[51] Berden GF, Althaus M, Verhulst FC. Major life events and changes in the behavioural functioning of children. The Journal of Child Psychology and Psychiatry. 1990,31(6):949-59.

[52] Feng DF. The influence of father absence on the mental development of rural left-behind children. Youth and Adolescent Studies.2019(04):13-18.

[53] Marceau K, Ram N, Houts RM. Individual differences in boys' and girls' timing and tempo of puberty: modeling development with nonlinear growth models. Development Psychology. 2011,47(5):1389-1409.

[54] Dou, C., Wei, Z., Jin, K., Wang, H., Wang, X., \& Peng, Z. (2015). Family and social environmental factors associated with aggression among Chinese adolescents. School Psychology Quarterly, 30(3), 421-430. https://doi.org/10.1037/spq0000103.

[55] Wang SH. The Research on the Cognitive Processing Bias among Resilience Level, Self-esteem and Emotional of Left-behind Children. Guangzhou University.2012.

[56] Zhang Fan. Mental Health and Resilience among Rural Left-behind Children in Three Gorges Areas. Chongqing Medical University.2013.

[57] Yang Yue. The Influence of Attachment Type and Staying Left or not on the Aggressiveness of Junior Middle School Students Under the Stressful Situation. Hebei Normal University.2018.

[58] Man Y, Mengmeng L, Lezhi L. The psychological problems and related influential factors of leftbehind adolescents (LBA) in Hunan, China: a cross sectional study. International Journal for Equity in Health. 2017,2,16(1):163.

[59] Chen C. The relationship among emotional maltreatment, aggression, resilience and peer relations within 4-6 grade pupils. Shenyang Normal University.2016.

[60] Song Ying. Setback Situation for Different Resilience Levels of Left-behind Junior Middle School Students' Influence on Aggression. Hebei Normal University.2018.

[61] Qi YM. The correlation research of the self-esteem, the resilience and the coping style of the rural junior middle school students. Northwest Normal University.2015.

[62] Han TW, Yang LS, Wang TZ, et al. Self-esteem status and its influencing factors among children in one rural area of Anhui Province in China. Chinese Journal of School Health. 2012,33(02):166-169.

[63] Liu QL, Zhou H, Yang Y, et al. Structural equation model analysis on psychological resilience of leftbehind children in rural areas in Sichuan Province. Journal of Hygiene Research. 2011,40(04):445-449. 
[64] Xu HS, Mu LL, Xie XL. Relationship of left-behind children's mental health, coping style, personality and self-efficacy. Journal of Hygiene Research. 2015,44(04):559-564.

Figures

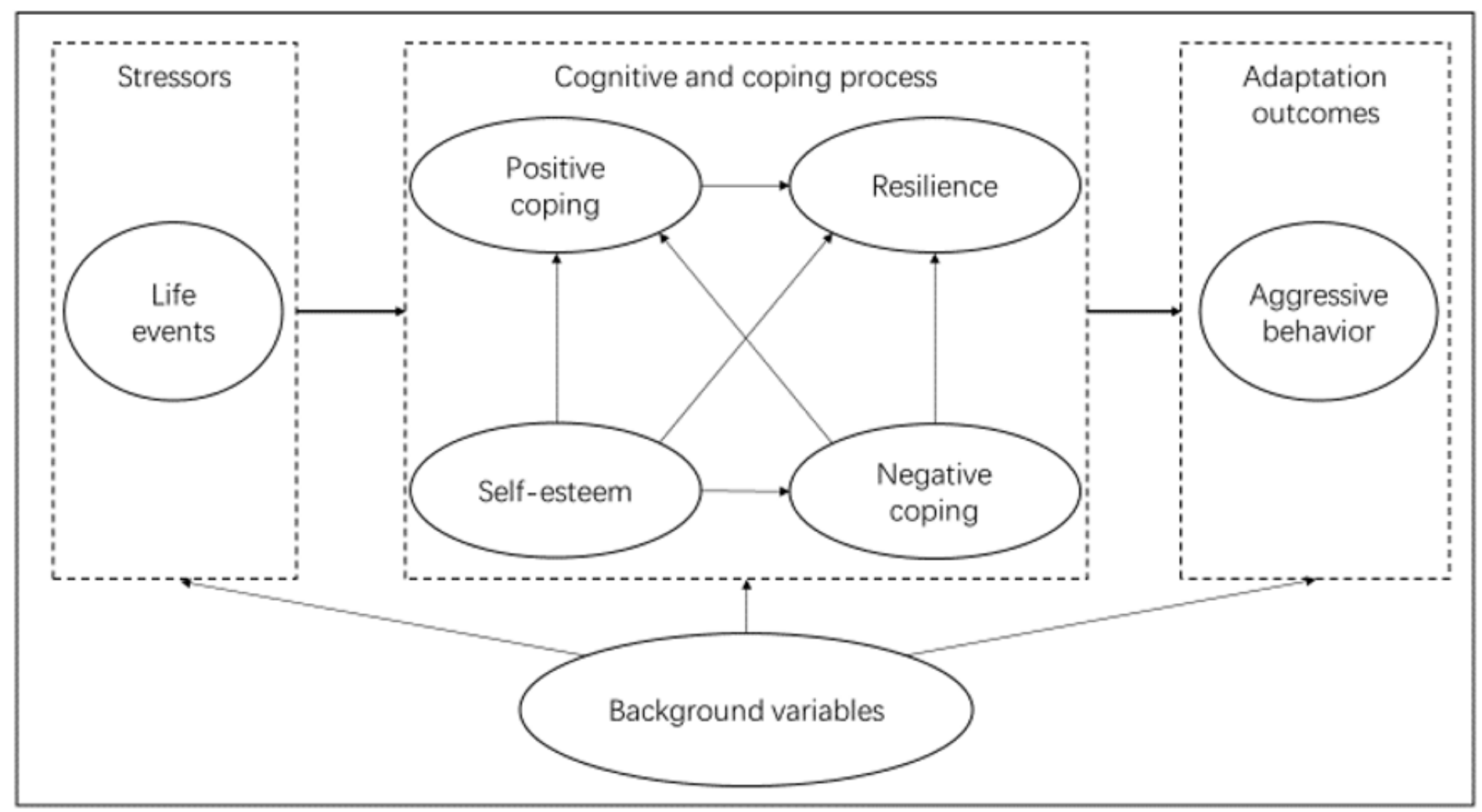

Figure 1

Hypothetical model A 


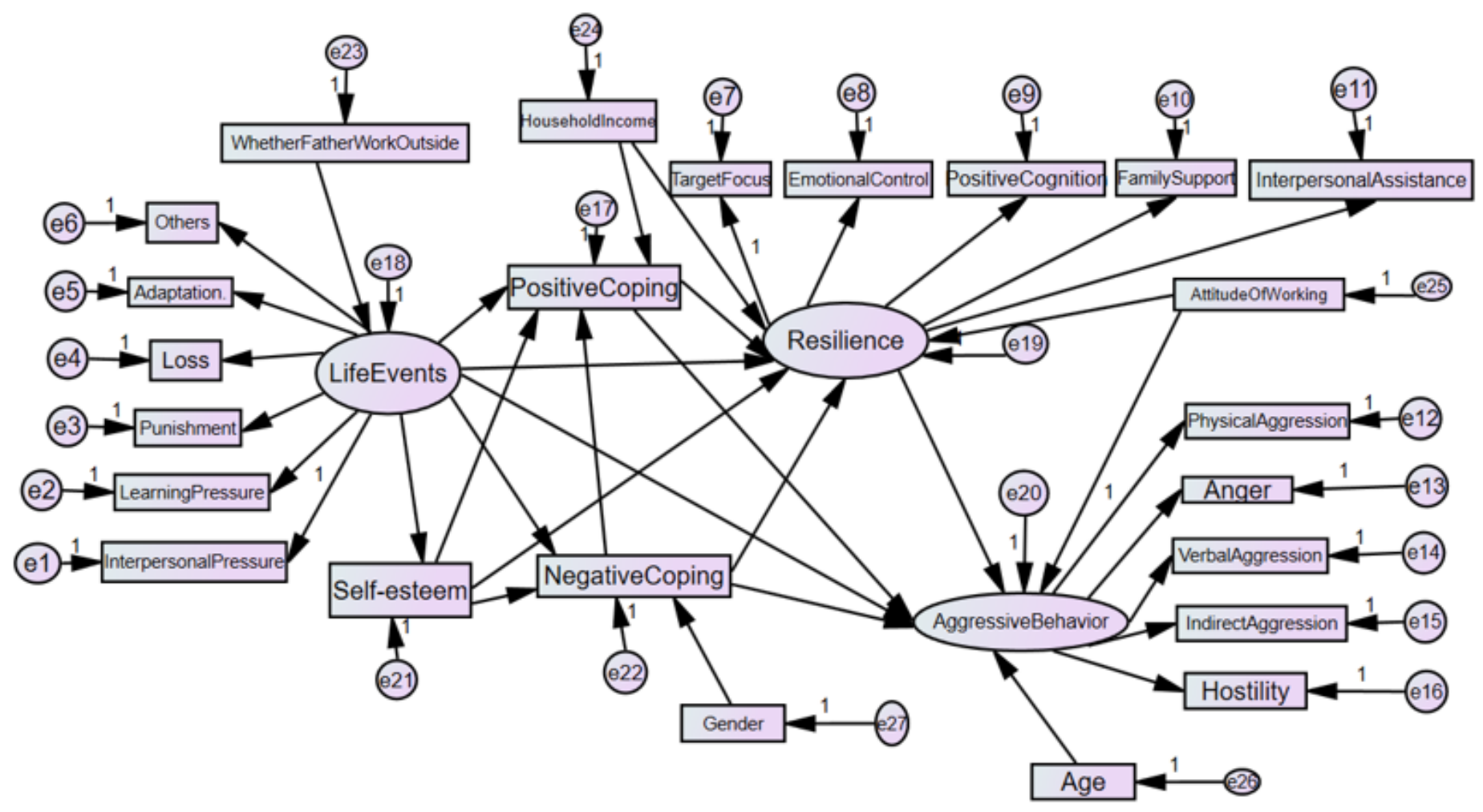

Figure 3

Hypothetical model B

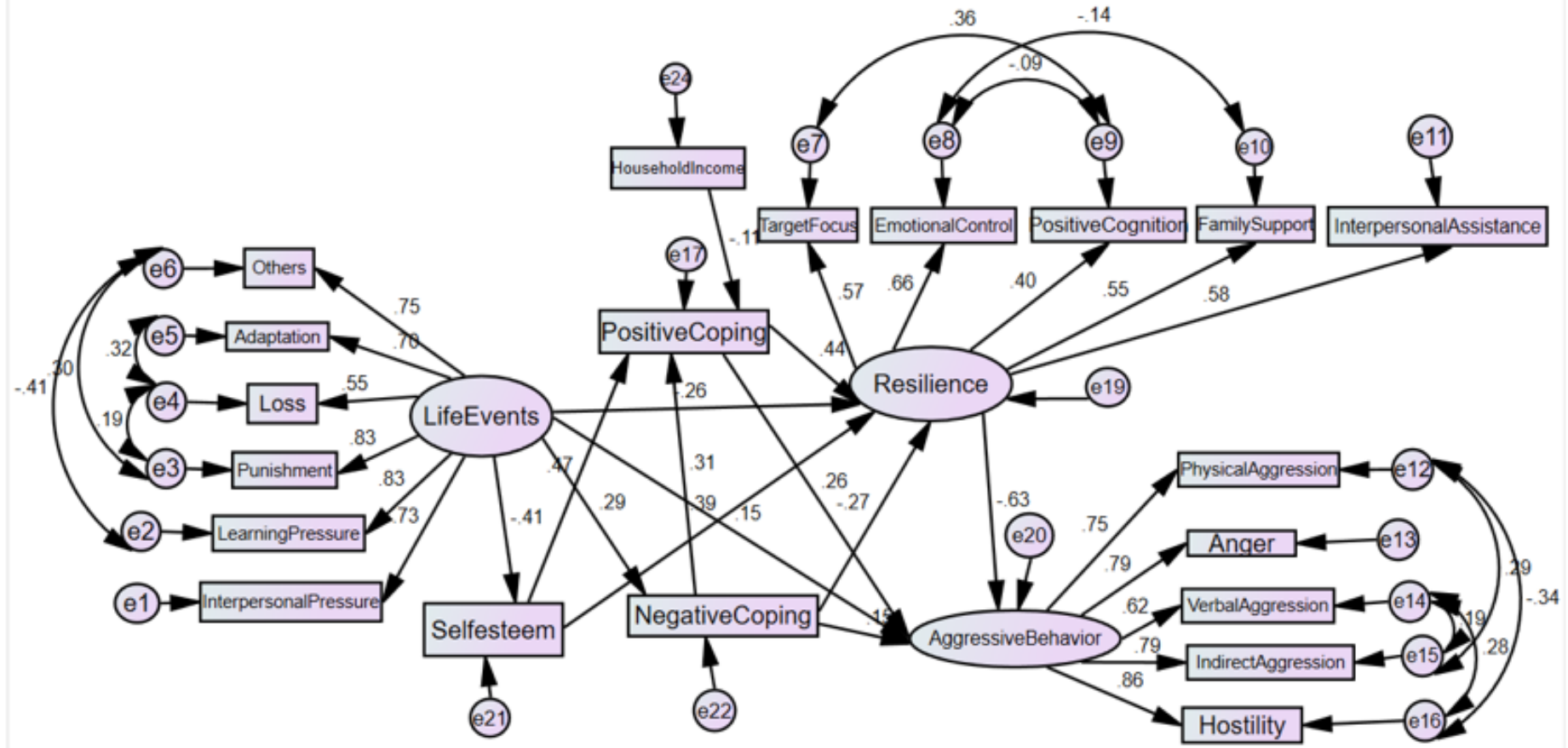


Figure 5

Modified model

Page 28/28 